\title{
Lyapunov-type inequalities for a class of even- order differential equations
}

\author{
Qi-Ming Zhang ${ }^{1 *}$ and Xiaofei $\mathrm{He}^{2}$
}

* Correspondence:

zhqm20082008@sina.com

'College of Science, Hunan

University of Technology, Zhuzhou, Hunan 412000, P.R. China

Full list of author information is

available at the end of the article

\section{Abstract}

We establish several sharper Lyapunov-type inequalities for the following even-order differential equation

$$
x^{(2 n)}(t)+(-1)^{n-1} q(t) x(t)=0 .
$$

These results improve some existing ones.

2000 Mathematics Subject Classification: 34B15.

Keywords: even-order, differential equation, Lyapunov-type inequality

\section{Introduction}

In 1907, Lyapunov [1] first established the Lyapunov inequality for the Hill's equation

$$
x^{\prime \prime}(t)+q(t) x(t)=0,
$$

which was improved to the following classical form

$$
(b-a) \int_{a}^{b} q^{+}(t) d t>4
$$

by Wintner [2] in 1951, if (1.1) has a real solution $x(t)$ such that

$$
x(a)=x(b)=0, \quad x(t) \not \equiv 0, \quad t \in[a, b],
$$

where $a, b \in \mathbb{R}$ with $a<b$, and the constant 4 cannot be replaced by a larger number, where and in the sequel $q^{+}(t)=\max \{q(t), 0\}$. Since then, there are many improvements and generalizations of (1.2) in some literatures. Especially, Lyapunov inequality has been generalized extensively to the higher-order linear equations and the linear Hamiltonian systems. A thorough literature review of continuous and discrete Lyapunov-type inequalities and their applications can be found in the survey article by Cheng [3]. Some other recent related results can be found in the articles [4-14].

We consider the even-order equation

$$
x^{(2 n)}(t)+(-1)^{n-1} q(t) x(t)=0,
$$

where $n \in \mathbb{N}, q(t)$ is a locally Lebesgue integrable real-valued function defined on $\mathbb{R}$. While $n=1$, the equation (1.4) reduces to the equation (1.2).

(c) 2012 Zhang and He; licensee Springer. This is an Open Access article distributed under the terms of the Creative Commons Attribution License (http://creativecommons.org/licenses/by/2.0), which permits unrestricted use, distribution, and reproduction in any medium, provided the original work is properly cited. 
For (1.4), there are several literatures having established some Lyapunov-type inequalities. For example, Yang [15,16] and Cakmak [17] have contributed to these interesting results. In the recent article [18], He and Tang improved and generalized the above results and obtained the following Lyapunov-type inequality.

Theorem 1.1. [18]Let $n \in \mathbb{N}$ and $n \geq 2, q \in L^{1}([a, b], \mathbb{R})$. If (1.4) has a solution $x(t)$ satisfying the boundary value conditions

$$
x^{(2 i)}(a)=x^{(2 i)}(b)=0, \quad i=0,1, \ldots, n-1, \quad x(t) \not \equiv 0, \quad t \in(a, b),
$$

then

$$
\int_{a}^{b} q^{+}(t)[(t-a)(b-t)]^{2} d t>\left(\frac{32}{45}\right)^{(1-\cos n \pi) / 4} \frac{2^{(n-2) / 2} 3^{n-1} 5^{(n-2) / 2}}{(b-a)^{2 n-5}} .
$$

In this article, motivated by the references [15-18], we attempt to establish some sharper Lyapunov-type inequalities for (1.4) under the same boundary value conditions of Theorem 1.1.

\section{Main results}

In the proof of our results, the following lemma is very important.

Lemma 2.1. [18]Assume that $x(t)$ is a continuous real-valued function on $[a, b], x(a)$ $=x(b)=0, x(t) \not \equiv$ ofor $t \in[a, b]$, and $x^{\prime \prime} \in L^{2}([a, b], \mathbb{R})$. Then

$$
|x(t)| \leq \frac{(t-a)(b-t)}{\sqrt{3(b-a)}}\left(\int_{a}^{b}\left|x^{\prime \prime}(s)\right|^{2} d s\right)^{1 / 2}, \quad \forall t \in[a, b] .
$$

In the meantime, in order to obtain Lemma 2.3 which also plays an important role in this article, we will apply the following inequality. See Lemma 2.2.

Lemma 2.2. [19]Assume that $f(t)$ and $f^{\prime}(t)$ are continuous on $[\alpha, \beta], f(\alpha)=f(\beta)$ and $\int_{\alpha}^{\beta} f(t) d t=0$. Then

$$
\int_{\alpha}^{\beta}|f(t)|^{2} d t \leq\left(\frac{\beta-\alpha}{2 \pi}\right)^{2} \int_{\alpha}^{\beta}\left|f^{\prime}(t)\right|^{2} d t .
$$

Lemma 2.3. Assume that $x(t)$ is a continuous real-valued function on $[a, b], x(a)=x$ $(b)=0, x(t) \not \equiv$ for $t \in[a, b]$, and $x^{\prime}, x^{\prime \prime} \in L^{2}([a, b], \mathbb{R})$. Then

$$
\begin{aligned}
& \int_{a}^{b}|x(t)|^{2} d t \leq \frac{(b-a)^{2}}{\pi^{2}} \int_{a}^{b}\left|x^{\prime}(t)\right|^{2} d t \\
& \int_{a}^{b}|x(t)|^{2} d t \leq \frac{(b-a)^{2}}{\pi^{2}} \int_{a}^{b}\left|x^{\prime \prime}(t)\right|^{2} d t .
\end{aligned}
$$

Proof. At first, we construct a function $f(t)$ as follows

$$
f(t)=\left\{\begin{array}{l}
x(t), \quad t \in[a, b] \\
-x(2 a-t), \quad t \in[2 a-b, a] .
\end{array}\right.
$$


Let $\alpha=2 a-b, \beta=b$. Since $x(a)=x(b)=0, x(a)=x(b)=0, x(t) \not \equiv 0$ for $t \in[a, b]$, and taking into account of the definition of $f(t)$, we can easily obtain that $\int_{a}^{b} f(t) d t=0$ and $f(\alpha)=f(\beta)$. Moreover, it is obvious that $f(t)$ and $f(t)$ are continuous on $[\alpha, \beta]$, since $x(t)$ is a continuous real-valued function on $[a, b]$. Hence, it follows from Lemma 2.2 that

$$
\int_{\alpha}^{\beta}|f(t)|^{2} d t \leq\left(\frac{2(b-a)}{2 \pi}\right)^{2} \int_{\alpha}^{\beta}\left|f^{\prime}(t)\right|^{2} d t
$$

Since

$$
\int_{\alpha}^{\beta}|f(t)|^{2} d t=\int_{2 a-b}^{a}|-x(2 a-t)|^{2} d t+\int_{a}^{b}|x(t)|^{2} d t=2 \int_{a}^{b}|x(t)|^{2} d t,
$$

and

$$
\int_{\alpha}^{\beta}\left|f^{\prime}(t)\right|^{2} d t=\int_{2 a-b}^{a}\left|-x^{\prime}(2 a-t)\right|^{2} d t+\int_{a}^{b}\left|x^{\prime}(t)\right|^{2} d t=2 \int_{a}^{b}\left|x^{\prime}(t)\right|^{2} d t
$$

it follows from (2.5), (2.6), and (2.7) that

$$
2 \int_{a}^{b}|x(t)|^{2} d t \leq 2\left(\frac{2(b-a)}{2 \pi}\right)^{2} \int_{a}^{b}\left|x^{1}(t)\right|^{2} d t
$$

which implies that the inequality (2.3) holds.

Next, we'll prove that the inequality (2.4) holds.

For convenience, we only consider the special case $a=0$. At this moment, the interval $[\alpha, \beta]$ reduces to $[-b, b]$. It follows from the construction of $f(t)$, that $f(t)$ is an odd function on $[-b, b]$, so we have $f(-t)=-f(t)$. Then, according to the definition of derivation, we have

$$
\begin{aligned}
f_{+}^{\prime}(-b) & =\lim _{t \rightarrow 0^{+}} \frac{f(t-b)-f(-b)}{t} \\
& =\lim _{t \rightarrow 0^{+}} \frac{-f(b-t)+f(b)}{t} \\
& =\lim _{t \rightarrow 0^{+}} \frac{f(b-t)-f(b)}{-t} \\
& =\lim _{s \rightarrow 0^{-}} \frac{f(b+s)-f(b)}{s} \\
& =f^{\prime}(b) .
\end{aligned}
$$

It follows from (2.9) that $f(\alpha)=f(\beta)$. Furthermore, we can easily obtain $\int_{\alpha}^{\beta} f^{\prime}(t) d t=0$ for the property that $f(t)$ is an odd function on $[\alpha, \beta]$. And the condition that $f^{\prime}(t)$ is continuous on $[\alpha, \beta]$ implies that $f^{\prime}(t)$ is continuous on $[\alpha, \beta]$, too. Then, by a similar method to the proof of (2.3) together with Lemma 2.2, we can obtain (2.4) immediately. 
For the other ordinary cases, i.e., $a \neq 0$, we only need to move the interval $[\alpha, \beta]$ evenly such that this interval symmetrizes about the origin. Then, similar to the proof of (2.9), we can verify the condition $f^{\prime}(\alpha)=f^{\prime}(\beta)$, and the other conditions in Lemma 2.2 are satisfied all the way. Hence, it also follows from Lemma 2.2 that (2.4) holds.

Theorem 2.4. Let $n \in \mathbb{N}, q \in L^{1}([a, b]$, $\mathbb{R})$. If (1.4) has a solution $x(t)$ satisfying the boundary value conditions (1.5), then

$$
\int_{a}^{b}|q(t)|^{2} d t \geq \frac{48 \pi^{4(n-1)}}{(b-a)^{4 n-1}} .
$$

Proof. Choose $c \in(a, b)$ such that $|x(c)|=\max _{t \in[a, b]}|x(t)|$. It follows from (1.5) that $x(a)=x(b)=0, x(a)=x(b)=0, x(t) \not \equiv 0$ for $t \in[a, b]$, which implies that $|x(c)|>$ 0 . Since (1.5), together with (1.4), Lemmas 2.1 and 2.3, we have

$$
\begin{aligned}
|x(c)|^{2} & \leq \frac{(b-a)^{3}}{48} \int_{a}^{b}\left|x^{\prime \prime}(t)\right|^{2} d t \\
& \leq \frac{(b-a)^{3}}{48}\left(\frac{(b-a)^{4}}{\pi^{4}}\right) \int_{a}^{n-1}\left|x^{b}(2 n)(t)\right|^{2} d t \\
& =\frac{(b-a)^{3}}{48}\left(\frac{(b-a)^{4}}{\pi^{4}}\right)^{n-1} \int_{a}^{b}|q(t)|^{2}|x(t)|^{2} d t .
\end{aligned}
$$

Since $|x(c)|>0$, divided the last inequality of (2.11) by $|x(c)|$, we can obtain (2.10).

By a similar method in the proof of Theorem 1.1, applying Lemmas 2.1 and 2.3, we can obtain the following result:

Theorem 2.5. Let $n \in \mathbb{N}$ and $n \geq 2, q \in L^{1}([a, b], \mathbb{R})$. If (1.4) has a solution $x(t)$ satisfying the boundary value conditions (1.5), then

$$
\int_{a}^{b} q^{+}(t)[(t-a)(b-t)]^{2} d t \geq \frac{3 \pi^{2 n-4}}{(b-a)^{2 n-5}} .
$$

Proof. From (1.5), multiplying (1.4) by $x(t)$ and integrating by parts over $[a, b]$, we have

$$
\int_{a}^{b} x^{(2 n)}(t) x(t) d t=(-1)^{n} \int_{a}^{b}\left|x^{(n)}(t)\right|^{2} d t .
$$

Combining (1.4) and (2.13), we have

$$
\int_{a}^{b} q(t)|x(t)|^{2} d t=\int_{a}^{b}\left|x^{(n)}(t)\right|^{2} d t .
$$

Case 1. If $n=2 m$ is an even number, then

$$
\int_{a}^{b} q(t)|x(t)|^{2} d t=\int_{a}^{b}\left|x^{(2 m)}(t)\right|^{2} d t .
$$


It follows from (1.5), (2.1), and (2.4) that

$$
|x(t)|^{2} \leq \frac{[(t-a)(b-t)]^{2}}{3(b-a)} \int_{a}^{b}\left|x^{\prime \prime}(s)\right|^{2} d s, \quad \forall t \in[a, b],
$$

and

$$
\int_{a}^{b}\left|x^{(2 i)}(t)\right|^{2} d t \leq \frac{(b-a)^{4}}{\pi^{4}} \int_{a}^{b}\left|x^{(2 i+2)}(t)\right|^{2} d t, \quad i=1,2, \ldots, m-1 .
$$

From (2.15), (2.16), and (2.17), we have

$$
\begin{aligned}
\int_{a}^{b} q^{+}(t)|x(t)|^{2} d t \leq & \int_{a}^{b} \frac{[(t-a)(b-t)]^{2}}{3(b-a)} q^{+}(t) d t \int_{a}^{b}\left|x^{\prime \prime}(t)\right|^{2} d t \\
& \leq \frac{1}{3(b-a)}\left(\frac{(b-a)^{4}}{\pi^{4}}\right) \int_{a}^{m-1} q^{+}(t)[(t-a)(b-t)]^{2} d t \int_{a}^{b}\left|x^{(2 m)}(t)\right|^{2} d t \\
& =\frac{(b-a)^{4 m-5}}{3 \pi^{4(m-1)}} \int_{a}^{b} q^{+}(t)[(t-a)(b-t)]^{2} d t \int_{a}^{b} q(t)|x(t)|^{2} d t \\
& \leq \frac{(b-a)^{4 m-5}}{3 \pi^{4(m-1)}} \int_{a}^{b} q^{+}(t)[(t-a)(b-t)]^{2} d t \int_{a}^{b} q^{+}(t)|x(t)|^{2} d t .
\end{aligned}
$$

Now, we claim that

$$
\int_{a}^{b} q^{+}(t)|x(t)|^{2} d t>0
$$

If (2.19) is not true, we have

$$
\int_{a}^{b} q^{+}(t)|x(t)|^{2} d t=0
$$

From (2.15) and (2.20), we have

$$
0 \leq \int_{a}^{b}\left|x^{(2 m)}(t)\right|^{2} d t=\int_{a}^{b} q(t)|x(t)|^{2} d t \leq \int_{a}^{b} q^{+}(t)|x(t)|^{2} d t=0,
$$

which implies that

$$
\int_{a}^{b}\left|x^{(2 m)}(t)\right|^{2} d t \equiv 0
$$

Then, from (2.22), we can obtain $x^{(2 m)}(t)=0$, for $t \in[a, b]$, which contradicts (1.5). So, (2.19) holds, and divided the last inequality of (2.18) by $\int_{a}^{b} q^{+}(t)|x(t)|^{2} d t$, we can obtain

$$
\int_{a}^{b} q^{+}(t)[(t-a)(b-t)]^{2} d t \geq \frac{3 \pi^{4(m-1)}}{(b-a)^{4 m-5}} .
$$


That is

$$
\int_{a}^{b} q^{+}(t)[(t-a)(b-t)]^{2} d t \geq \frac{3 \pi^{2 n-4}}{(b-a)^{2 n-5}} .
$$

Case 2. If $n=2 m+1$ is an odd number, then

$$
\int_{a}^{b} q(t)|x(t)|^{2} d t=\int_{a}^{b}\left|x^{(2 m+1)}(t)\right|^{2} d t .
$$

In one hand, it follows from (1.5), (2.1), and (2.4) that (2.16) and (2.17) hold. In the other hand, since $x^{(2 m)}(a)=x^{(2 m)}(b)=0$, it follows from (2.3) that

$$
\int_{a}^{b}\left|x^{(2 m)}(t)\right|^{2} d t \leq \frac{(b-a)^{2}}{\pi^{2}} \int_{a}^{b}\left|x^{(2 m+1)}(t)\right|^{2} d t .
$$

Hence, combining (2.16), (2.17), (2.25) with (2.26), we have

$$
\begin{aligned}
\int_{a}^{b} q^{+}(t)|x(t)|^{2} d t \leq & \int_{a}^{b} \frac{[(t-a)(b-t)]^{2}}{3(b-a)} q^{+}(t) d t \int_{a}^{b}\left|x^{\prime \prime}(t)\right|^{2} d t \\
& \leq \frac{1}{3(b-a)}\left[\frac{(b-a)^{4}}{\pi^{4}}\right]^{m-1} \int_{a}^{b} q^{+}(t)[(t-a)(b-t)]^{2} d t \int_{a}^{b}\left|x^{(2 m)}(t)\right|^{2} d t \\
& \leq \frac{b-a}{3 \pi^{2}}\left[\frac{(b-a)^{4}}{\pi^{4}}\right]^{m-1} \int_{a}^{b} q^{+}(t)[(t-a)(b-t)]^{2} d t \int_{a}^{b}\left|x^{(2 m+1)}(t)\right|^{2} d t \\
& =\frac{(b-a)^{4 m-3}}{3 \pi^{4 m-2}} \int_{a}^{b} q^{+}(t)[(t-a)(b-t)]^{2} d t \int_{a}^{b} q(t)|x(t)|^{2} d t \\
& \leq \frac{(b-a)^{4 m-3}}{3 \pi^{4 m-2}} \int_{a}^{b} q^{+}(t)[(t-a)(b-t)]^{2} d t \int_{a}^{b} q^{+}(t)|x(t)|^{2} d t
\end{aligned}
$$

Since (2.19), divided the last inequality of (2.27) by $\int_{a}^{b} q^{+}(t)|x(t)|^{2} d t$, we can obtain

$$
\int_{a}^{b} q^{+}(t)[(t-a)(b-t)]^{2} d t \geq \frac{3 \pi^{4 m-2}}{(b-a)^{4 m-3}} .
$$

That is

$$
\int_{a}^{b} q^{+}(t)[(t-a)(b-t)]^{2} d t \geq \frac{3 \pi^{2 n-4}}{(b-a)^{2 n-5}} .
$$

It follows from (2.24) and (2.29) that (2.12) holds.

Remark 2.6. In view of the forms of the two inequalities (1.6) and (2.12), we can easily find that inequality (2.12) is simpler than (1.6). Moreover, by using the method of induction, we can verify that inequality (2.12) is sharper than inequality (1.6). 
Corollary 2.7. Let $n \in \mathbb{N}$ and $n \geq 2, q \in L^{1}([a, b], \mathbb{R})$. If (1.4) has a solution $x(t)$ satisfying the boundary value conditions (1.5), then

$$
\int_{a}^{b} q^{+}(t) d t \geq \frac{48 \pi^{2 n-4}}{(b-a)^{2 n-1}} .
$$

\section{Author details}

${ }^{1}$ College of Science, Hunan University of Technology, Zhuzhou, Hunan 412000, P.R. China ${ }^{2}$ College of Mathematics and Computer Science, Jishou University, Jishou 416000, Hunan, P.R. China

\section{Authors' contributions}

QZ carried out the theoretical proof and drafted the manuscript. XH participated in the design and coordination. Both of the two authors read and approved the final manuscript.

\section{Competing interests}

The authors declare that they have no competing interests.

\section{Received: 19 October 2011 Accepted: 12 January 2012 Published: 12 January 2012}

\section{References}

1. Liapunov, AM: Problème général de la stabilité du mouvement (Russian). Ann Fac Sci Univ Toulouse. 2, 203-407 (1907)

2. Hartman, P, Wintner, A: On an oscillation criterion of Lyapunov. Am J Math. 73, 885-890 (1951). doi:10.2307/2372122

3. Cheng, SS: Lyapunov inequalities for differential and difference equations. Fasc Math. 23, 25-41 (1991)

4. He, Z: Existence of two solutions of $m$-point boundary value problem for second dynamic equations on time scales. J Math Anal Appl. 296, 97-109 (2004). doi:10.1016/j.jmaa.2004.03.051

5. Jiang, LQ, Zhou, Z: Lyapunov inequality for linear Hamiltonian systems. J Math Anal Appl. 310, 579-593 (2005)

6. Kwong, MK: On Lyapunov's inequality for disfocality. J Math Anal Appl. 83, 486-494 (1981). doi:10.1016/0022-247X(81) $90137-2$

7. Lee, C, Yeh, C, Hong, C, Agarwal, RP: Lyapunov and Wirtinger inequalities. Appl Math Lett. 17, 847-853 (2004). doi:10.1016/.aml.2004.06.016

8. Pachpatte, BG: On Lyapunov-type inequalities for certain higher order differential equations. J Math Anal Appl. 195, 527-536 (1995). doi:10.1006/jmaa.1995.1372

9. Tang, XH, Zhang, M: Lyapunov inequalities and stability for linear Hamiltonian systems. J Diff Eqs. 252, 358-381 (2012). doi:10.1016/j.jde.2011.08.002

10. Wang, X: Stability criteria for linear periodic Hamiltonian systems. J Math Anal Appl. 367, 329-336 (2010). doi:10.1016/j. jmaa.2010.01.027

11. Wang, X: Lyapunov type inequalities for second-order half-linear differential equations. J Math Anal Appl. 382, 792-801 (2011). doi:10.1016/j.jmaa.2011.04.075

12. Yang, X, Kim, Y, Lo, K: Lyapunov-type inequalities for a class of quasilinear systems. Math Comput Model. 53, 1162-1166 (2011). doi:10.1016/.jmcm.2010.11.083

13. Zhang, Q, Tang, XH: Lyapunov inequalities and stability for discrete linear Hamiltonian system. Appl Math Comput. 2 574-582 (2011)

14. Zhang, Q, Tang, XH: Lyapunov inequalities and stability for discrete linear Hamiltonian systems. J Diff Equ Appl. (in press)

15. Yang, X: On inequalities of Lyapunov type. Appl Math Comput. 134, 293-300 (2003). doi:10.1016/50096-3003(01)00283-1

16. Yang, X: On Liapunov-type inequality for certain higher-order differential equations. Appl Math Comput. 134(2-3), 307-317 (2003). doi:10.1016/50096-3003(01)00285-5

17. Cakmak, D: Lyapunov-type integral inequalities for certain higher order differential equations. Appl Math Comput. 216, 368-373 (2010). doi:10.1016/j.amc.2010.01.010

18. He, X, Tang, XH: Lyapunov-type inequalities for even order differential equations. Commun Pure Appl Anal. 11(2), 465-473 (2012)

19. Almansi, E: Sopra una delle esperienze di Plateau (Russian). Ann Mat Pura Appl. 12, 1-17 (1905)

doi:10.1186/1029-242X-2012-5

Cite this article as: Zhang and He: Lyapunov-type inequalities for a class of even-order differential equations.

Journal of Inequalities and Applications 2012 2012:5. 